\title{
Rural Road Maintenance in Madagascar The GENIS project
}

\author{
Alexis Tsoukiàs(*), Herimandimbiniaina Ralijaona(+) \\ (*) CNRS - LAMSADE, Université Paris Dauphine, France \\ tsoukias@lamsade.dauphine.fr \\ (+) AGETIPA, Madagascar \\ herimandimbiniaina@agetipa.mg
}

\begin{abstract}
The paper reports a real world decision aiding process concerning rural road maintenance in Madagascar. The issue arises within AGETIPA, the National Agency in charge of conducting Public Works in Madagascar, and can be summarised as a problem of resource allocation to a number of competitive projects. The problem has been modeled using multiple criteria and a classification procedure under two objectives: make the most rational use of the limited available resources and promote participation and commitment of the local actors in the maintenance process. The project is part of an on-going partnership between the LAMSADE and AGETIPA aiming to enhance Decision Support Capacity within AGETIPA.
\end{abstract}

\section{Introduction}

This is a paper on a real case study concerning decision support issues arising from the problem of improving the rural road maintenance activity in Madagascar. This is one among the regular activities of AGETIPA and the occasion to focus the attention on it was given within the GENIS project.

AGETIPA (Agence d'Execution des Travaux d'Intérêt Public) is a non profit Agency established in 1993 by the Government of Madagascar and the consulting and executive companies working in the sector of the public work in Madagascar. Since 1994 it is responsible for the executive follow up of most of the public works taking place in Madagascar, the development projects as well as in charge of capacity building in this sector. It is a member of AFRICATIP the African Association of Public Work Agencies (there are 18 such agencies over the continent). Besides its activities in executive support AGETIPA provides technical support to other Institutions and Government bodies, training for the staff of the consulting 
and construction companies and recently develops also Decision Support Activities.

The GENIS project (Gestion des Routes par Niveau de Service) has been proposed by the Transportation Minister in collaboration with the World Bank in order to improve rural road maintenance. A pilot study area has been selected, the greater Antananarivo urban and peri-urban area. The principal aim of the project is to contribute, to the improvement of accessibility from rural areas to markets, social services and economic activities, thus increasing income for rural population, improving their life conditions and ultimately reducing poverty. The implementation of the GENIS project should:

- satisfy the needs of the users in terms of necessary physical conditions of the roads to be maintained;

- the service level of the roads to be considered should be maintained or improved. The principal decision problem within the GENIS project is to establish which roads should be considered first in order to maximise the positive economic, social and environmental impact.

While trying to formalise the problem on how to make any decisions concerning priorities in rural road maintenance the CEO of AGETIPA realised that:

1. there were no internal resources (at that time) able to follow a decision support problem (not only the specific one);

2. there was a clear necessity to re-think the whole evaluation model as far as public works financing was concerned, at least in the case of rural roads in Madagascar.

Although evaluating public projects in economical mature societies can be considered as a typical cost-benefit analysis (the reader should note that this is an approach strongly argued in the literature; see [1], [2], [4], [5], [6], [8], [9], [11], [14]), this is much more difficult in the case of countries under development. Deciding to fund a rural road maintenance project can be of vital importance for a local community and is basically a political decision which needs to be legitimated, explained and possibly negotiated. Behind such decisions there is a whole formal or informal process involving the participation of the local community and thus influencing also the future of this young democracy.

On that basis AGETIPA considered the hypothesis of involving a Decision Analysis expert with the aim: on the one hand to help conducting the GENIS 
project and on the other hand to introduce and enhance within AGETIPA a Decision Support capacity. This is how the first author has been involved in the project.

The presentation of this case study will follow the scheme presented in [15] in order to emphasise in a structured way the different cognitive artifacts produced in the decision aiding process. More precisely we will try to describe the:

- problem situation; who are the actors involved, what are their concerns and what are their commitments?

- problem formulation; on what the decision process focusses, under which points of view (available and/or requested knowledge) and for what precise purpose?

- evaluation model; what are the elements composing such a model (set of alternatives, attributes and their scales, criteria, uncertainties and procedures to be used)?

There will be no final recommendation to talk about, since the study concerned the construction of a method to apply in several different problem situations repeatedly. There has been a pilot study though for which recommendations have been formulated, but this was basically a validation activity.

The objectives of this study can be thus summarised.

- provide a methodology for handling the rural road network maintenance problem;

- develop a decision aiding methodology for similar problems;

- create decision support capacity within AGETIPA with respect both to "internal" and "external" clients.

The paper is thus organised as follows. In Section 2 we present the Problem Situation. In Section 3 we present the Problem Formulation, while in Section 4 we present the Evaluation Model(s) used in order to handle the problem as formulated in Section 3. In Section 5 we present the pilot study conducted in order to validate the method suggested. In Section 6 we present the feedback obtained after two years of using this method in rural road maintenance in Madagascar. Section 7 concludes the paper. 


\section{Problem Situation}

The maintenance and improvement of the rural road network in Madagascar is a vital project for the population living in these areas both for economic reasons (improve accessibility to land and markets) and social ones (improve accessibility to schools and health centres as well as improve communication among remotely located communities).

The result is an extremely high demand for rural road maintenance. In front of that the availability of funds to dedicate to this purpose is rather limited and largely depending from external donors and financial institutions such as the World Bank. The basic problem arises from this scarce availability of funds: given the annual spending of the national government for rural road maintenance where should be given the priority?

The demand for maintenance generates locally from communities and their local government which for simplicity we are going to represent by the local mayor. At the national level the management of the process is delegated by the government to AGETIPA who is expected to establish within the GENIS project the priorities and thus decide every year which are the projects that can be satisfied. However, in managing this problem AGETIPA is expected to pursue a precise policy:

- on the one hand maximise the positive impact of the policy in terms of benefiting population, economic activities impacted and general improvement of the whole road network of the island in terms of "level of service" guaranteed;

- on the other hand promote the participation and active implication of the local communities and their representatives as well as of possibly existing economic actors both to the decision and to the implementation process.

Under such a perspective, the accountability of the decision process is extremely important. It is important to explain why a project has been rejected AND under which conditions would have been accepted (or could actually be accepted).

The traditional approach used until the time of this study aiming at calculating the overall economic return of each project (seen as a public investment) seems inadequate with respect to the above policy for essentially two reasons:

- the difficulty to take into account qualitative information in order to establish an economic return (not all impacts of such projects have a clear economic dimension);

- the impossibility to establish a negotiation table with the local actors: indeed the accountability of decisions based on purely economic reasons is extremely difficult (at least with respect to precisely the local actors which are expected to be 
implied).

It should also be emphasised that the "client" (the government) considers very important to allow transparent negotiations with the local actors where the reasons for which projects submitted were rejected should be clear. A rural road can be of vital importance for a local community and rejecting its maintenance project can induce severe consequences.

We can summarise the problem situation as follows.

- The actors involved in the decision process (the stakeholders) are the National Government, AGETIPA, the local actors (economic, social and political), the population itself, fund raising institutions, international donors and financial institutions.

- The objects/concerns of such actors include: the rural roads network and the specific maintenance projects for given segments of the network, the funds available for maintenance at the National level, any local funds available, the decision process itself, the negotiation capacity, the local actors implication and commitment, the long term evolution of the whole road network of the island, the long term impact of this process on regional planning at the National level.

- The resources committed in the decision process include the maintenance funds, the local knowledge of the rural road network and the social and economic needs of the local communities, the satellite images of the rural areas, other information (statistics) about the areas crossed by the rural roads network, the technical knowledge about planning and road maintenance.

Decision Aiding is thus expected to:

- convince AGETIPA that the projects chosen will have the maximum positive impact;

- be inserted within a long term effort of improving regional planning and more precisely road maintenance;

- be accountable with respect to the National government, the local actors and the international donors and financial institutions;

- allow a transparent decision process enabling negotiations with the local actors. 


\section{Problem Formulation}

The problem situation as previously described has been discussed within AGETIPA together with their advisor (the first author). Several hypotheses have been considered. In order to establish a precise problem formulation the following points have been fixed:

- the results of the model have to be easily explainable and give an immediate intuitive idea of the decision;

- the results of the model have to be consistent with the long term government policy in road network improvement;

- the model has to take into account the local actors' commitment.

On this basis the following problem formulation has been adopted: Classify the submitted projects into three classes: accepted, rejected, negotiable, taking into account the social-economic impact of the projects, the local actors' commitment and the cost. More precisely the problem formulation contains the following elements.

$\mathbb{A}$ The actions to be considered by the decision aiding process should be the precise rural road maintenance projects expressed and submitted by the local communities. More precisely the projects are established through a consultation process organised by FIFTAMA (the Association of the mayors of the towns surrounding Antananarivo). Some basic conditions are expected to be met in order to be eligible to apply:

- the roads to be maintained need to be already inserted within the Provincial Transportation Plan established in 2004;

- the roads need to already exist in "acceptable" physical conditions and to mobilise local resources;

- the roads need to be part of transportation axes linking the communities between them, axes identified in local development plans and have to intersect the national road network.

$\mathbb{V}$ There are basically three points of view to take into account for assessing the various projects:

- the first one concerns the "commitment" of the local actors to the maintenance effort. The idea here is that the local actors should consider the rural road maintenance as one of their major commitments and that they should feel responsible for such a resource;

- the second one concerns the "social-economic" impact of each project for 
the local community. As already mentioned the rural road maintenance is considered as a key element of an overall policy aiming to sustain local development, reduce isolation, increase the economic, social and cultural exchanges and integrate the whole road network of the island. Proposed projects therefore need to demonstrate that they improve both internal accessibility (to key areas of the community) and external accessibility (to the national road network, both in quantity and in quality. Besides, priority should be given to areas with high population and/or economic density (priority expressed by the national government).

- the third one is the "cost", including both short term and long term financial costs as well as negative environmental and social impacts.

$\Pi$ The problem statement adopted in this case is to classify the projects in three ordered categories:

- accepted, the ones that meet all requirements and result at the top of all priorities in "all" dimensions (the ones for which the positive decision appears fully legitimate);

- rejected, the ones that definitely cannot be accepted resulting at the bottom of the priorities in "all" dimensions (the ones for which the negative decision appears fully legitimate);

- negotiable, the ones who could be accepted in case one or more dimensions could be improved.

\section{Evaluation Model}

The evaluation model has been conceived in a straightforward implementation of the problem formulation. It has been constructed in two steps. The first has been a generic model suggested by AGETIPA which has been refined after discussion with the advisor in order to fulfill basic conditions of coherence of the model (for a discussion about the coherence of a model the reader can see [2], [16]). The second has been a more precise version adapted to the precise issues raised by the GENIS project. The presentation which follows concerns the model as conceived before the first round of applications (there is no mention to uncertainty issues since it was considered that there was no such problem presently). In the feedback section we present how the model has been modified. 


\subsection{Alternatives}

The alternatives to be evaluated are considered to be the projects submitted by the local communities. Such projects concern precise segments of the rural roads network which can normally be identified on the satellite images of the whole island (data to which AGETIPA has access). Such projects concern already existing segments of the network and not new connections to build. It is assumed that for each project presented it is possible to identify the local community submitting it (a single administration or a group of administrations).

\subsection{Dimensions and Measurement Scales}

The structure of dimensions results from the analysis of the three points of view introduced in the problem formulation.

1. The "Local Commitment" dimension decomposes to two attributes: public and private participation and is measured considering the level of financial involvement of the local community and/or the private actors to both the immediate costs of the project as well as the subsequent maintenance costs. Practically we get two figures expressed in percentages.

2. The "social-economic impact" dimension decomposes to four attributes:

- Internal accessibility. On its turn this attribute results taking into account four sub-attributes:

- the number of social, cultural and administrative centres served by the project (schools, churches, townhalls etc.);

- the agricultural area served by the project (measured in ha);

- the number of "economic activities centres" served (such as markets, industries etc.);

- the "level of service" presently offered by the road under consideration. The "level of service" is established considering three types of data:

- seasonal availability;

- maximum speed;

- traveling comfort;

- External accessibility. On its turn this attribute results taking into account four sub-attributes:

- number of intersections of the proposed road maintenance project 
with the national road network;

- state of the above intersections;

- volume of traffic on the road (including the transit traffic);

- existence of alternatives and/or shortcuts in case of major impediments.

- Population density. Measured by number of people living in the area by square $\mathrm{km}$.

- Economic density. Measured by the average taxes perceived in the area by the number of people living in the area.

3. The "cost" dimension is represented by a single attribute representing the cost of the project submitted taken into account both the immediate costs and the subsequent maintenance costs. No further environmental or social costs have been considered at this level of the study.

The information concerning the above attributes have been collected from the satellite images to whom AGETIPA has access, from the census information of Madagascar and finally from direct visits in the interested area in order to double check doubtful information.

\subsection{Criteria}

The set of criteria results in an hierarchy as can be shown in figure 1. Leaves have simple preference models resulting from the client's values. Intermediate "parent" nodes have preference models resulting from aggregating the preferences expressed on the "son" nodes.

In the following we are going to present the preference models starting from the leaves of the criteria tree (the ones which have no "son" nodes).

1. Public Participation will be represented through a simple model, such that $x \succeq y$ iff $p u(x) \geq p u(y)$, where $p u(x)$ is the percentage of funds contributed by the local community to the project.

2. Private Participation will be represented through a simple model, such that $x \succeq y$ iff $\operatorname{pr}(x) \geq \operatorname{pr}(y)$, where $\operatorname{pr}(x)$ is the percentage of funds contributed by private actors, possibly interested within the local community, to the project. 


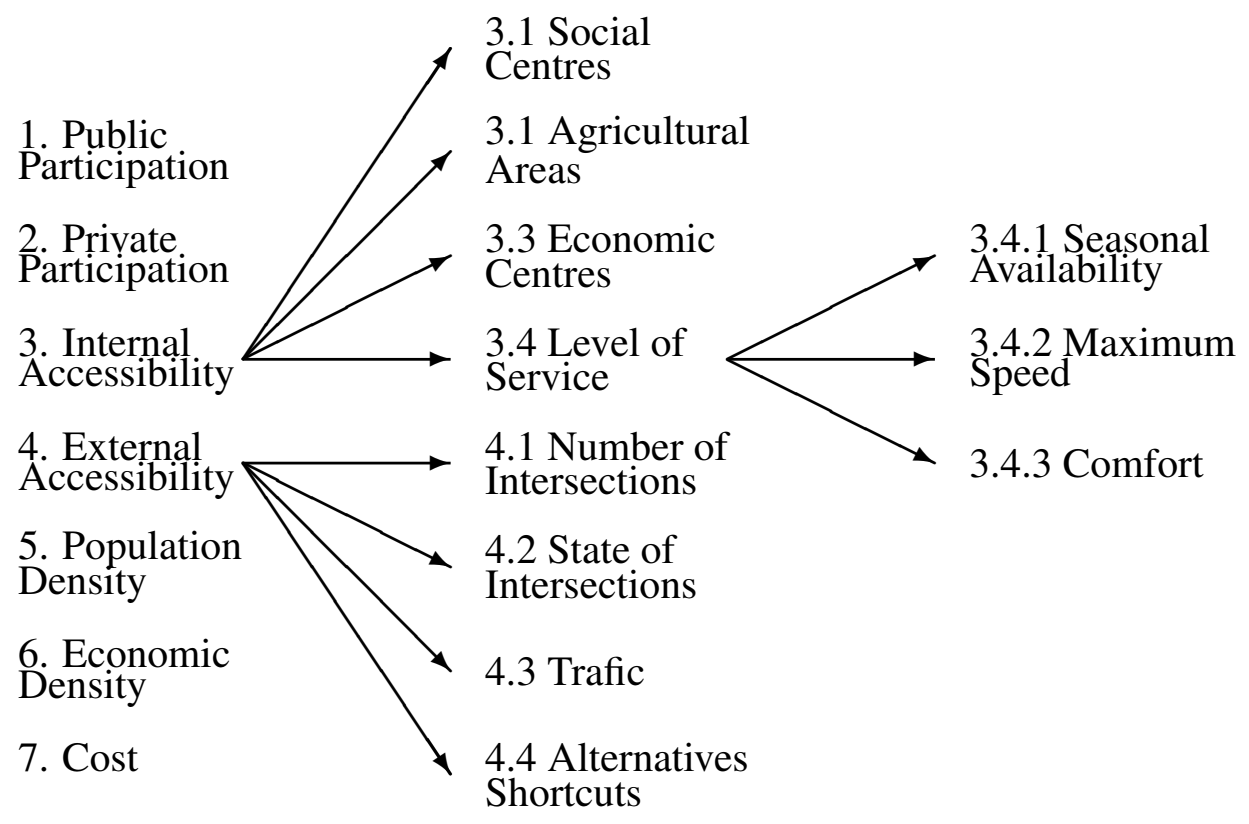

Figure 1: The set of criteria

5. Population Density will be represented through a simple model, such that $x \succeq y$ iff $d(x) \geq d(y)$, where $d(x)$ is the number of habitants by square $\mathrm{km}$ in the area interested by the project.

6. Economic Density will be represented through a simple model, such that $x \succeq y$ iff $e(x) \geq e(y)$, where $e(x)$ is the average taxes perceived for each inhabitant in the area interested by the project.

7. Cost will be represented through a simple model such that $x \succeq y$ iff $c(x) \leq$ $c(y)$ where $c(x)$ is the cost (immediate and subsequent maintenance) of the project.

In order to present the model of criterion 4 we need to present the preference models of nodes 4.1 to 4.4 .

4.1 Number of Intersections simply takes into account how many intersections the project has with the national road network. There are three possible states (1, 2 or 3 intersections) and the more intersections the better it is (the case of no intersection is no considered since it is a reason of exclusion). 
4.2 State of Intersections is a qualitative judgement: 0 for a bad condition, 1 for acceptable condition and 2 for good condition.

4.3 Traffic measures the volume of traffic as resulting from the official statistics (considering a market day and using the usual coefficients for "homogeneous vehicles", HV). Three levels are considered: less than $500 \mathrm{HV}$ daily, less than $1000 \mathrm{HV}$ daily and more than $1000 \mathrm{HV}$ daily. The more trafic registered the more important is the project.

4.4 Alternatives and/or Shortcuts takes into account the presence of such routes. Three possible values are considered: no alternatives and shortcuts, either an alternative or a shortcut, both alternatives and shortcuts.

Criterion 4 is thus established on a discrete scale with values 1 (bad), 2 (acceptable), 3 (good). The procedure for assigning a value to a precise alternative is based on an ordered classification procedure of the type ELECTRE TRI (a simplified version based only on simple majority rules, for more details see for instance [3], [12]). The model is presented in figure 2. Projects whose profile is (by majority) on the left of the frontier $\langle 1,1,500, N N\rangle$ get the value 1, projects whose profile is (by majority) on the right of the frontier $\langle 2,2,1000, Y Y\rangle$ get the value 3 , the rest get the value 2 .

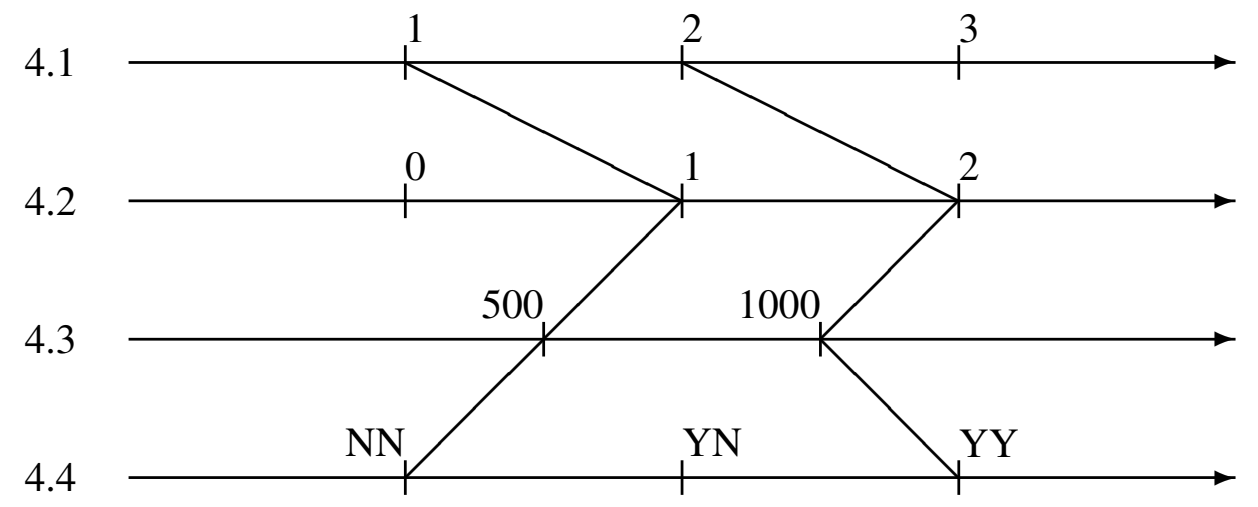

Figure 2: Model of criterion 4

In order to establish the model of criterion 3 we first need to establish the model of node 3.4. This is shown in figure 3. Projects are noted on a four valued scale (1-4) resulting from an explicit enumeration of the 48 combinations of the 11 values on the nodes 3.4.3 (comfort I: unacceptable, A: acceptable, M: medium, 
B: good), 3.4 .2 (maximum speed, less than $10 \mathrm{~km} / \mathrm{h}, 10-30 \mathrm{~km} / \mathrm{h}, 30-50 \mathrm{~km} / \mathrm{h}$, more than $50 \mathrm{~km} / \mathrm{h}$ ), 3.4 .1 (seasonal availability: less than 6 months annually, from 6 to 9 months annually, more than 9 months annually).

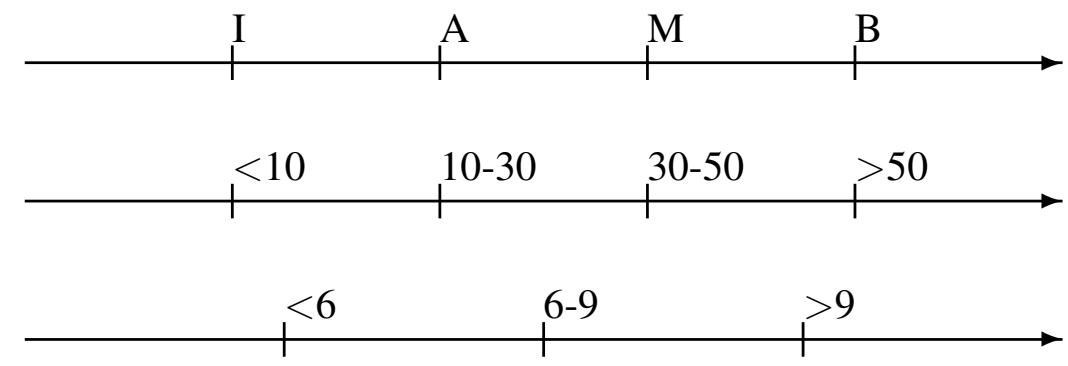

Figure 3: Model of node 3.4

Criterion 3 is also constructed putting together the four assessments expressed on nodes 3.1, 3.2, 3.3 and 3.4. The model of node 3.4 has been presented previously. We now present the models of nodes 3.1, 3.2 and 3.3.

3.1 There are three possible values: less than 5 social centres served, 5 to 10 and more than 10 social centres served. In counting such social centres we count principally schools, hospitals (and other health centres) churches and administration centres.

3.2 We take into account the land used (or potentially used) for agricultural purposes. This is measured in hectares.

3.3 There are three possible values: less than 5 economic centres served, 5 to 10 and more than 10 economic centres served. In counting such centres we count principally markets and any other economic activity which may attract mobility within the community.

Criterion 3 is thus established on a discrete scale with values $\mathrm{M}$ (bad), L (borderline), A (acceptable), B (good). The procedure for assigning a value to a precise alternative is based on an ordered classification procedure of the type ELECTRE TRI (a simplified version based only on simple majority rules, as for criterion 4). The model is presented in figure 4. Projects whose profile is (by majority) on the left of the frontier $\langle<5,20,<5,3\rangle$ get the value M, projects whose profile is (by majority) on the right of the frontier $\langle>10,100,>10,1\rangle$ get the value B. Projects who are on the right of the lowest profile, but lower than the profile $\langle 5-10,50,5-10,2\rangle$ get the value $\mathrm{L}$, the rest get the value $\mathrm{A}$. 
3.1

3.2

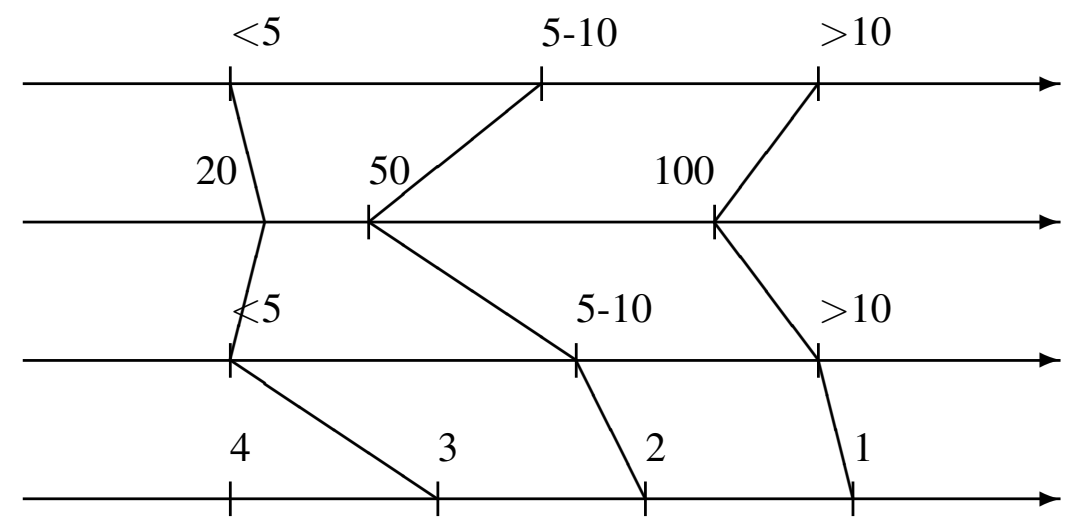

Figure 4: Model of criterion 3

\subsection{Aggregation Procedure}

The overall aggregation procedure was aimed at assigning each project to one among the following classes: accepted, negotiable, rejected. The procedure once again is a simplified version of the ELECTRE TRI method. The final model can be seen in figure 8. A brief description of the method is provided in Appendix A. In the pilot study a "pessimistic" assignment procedure has been adopted. Generally speaking we adopted a very simple ordinal sorting procedure mostly in order to ease the explanations for each assignment decision and thus the discussion which followed.

\section{Pilot Study}

The proposed method has been tested in a pilot study concerning an area at the Nord East of the Great Antananarivo Metropolitan Area. This area can be seen in figure 5.

Data have been collected from satellite images (to which AGETIPA has access), from the National Statistics office and from direct visits in the area under study. A data base with all relevant information has been thus established. A typical record of this data base can be seen in figure 6 .

Four maintenance projects have been selected in order to be evaluated as a test for the method. These were at the moment of the study under discussion, but a basic consensus was already reached, although not using a formal evaluation tool. For this reason they seemed to be an interesting benchmark. The values of the four 


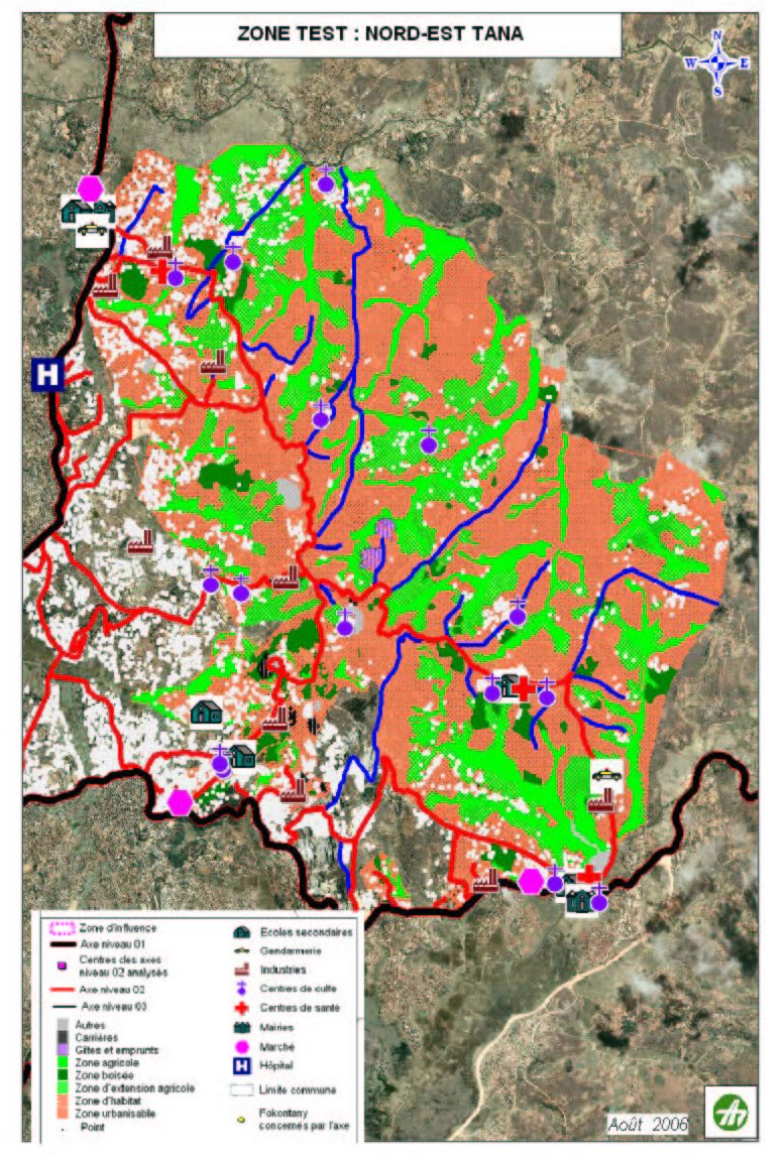

Figure 5: Map of the pilot study area: Nord East of Antananarivo

alternatives together with the chosen profiles for the three categories (to finance, to negotiate, to reject) can be seen in figure 7 .

The classification model specifying the shape of the three categories can be seen in figure 8

In order to complete the model we need to establish the "importance parameters", that is the importance of each criterion for establishing the class of each alternative. While in the intermediate nodes of the hierarchy a simple majority rule has been adopted, the final model needs to take more precisely into account the importance that the decision maker desires to associate to each criterion (as a manifestation of a certain policy). 


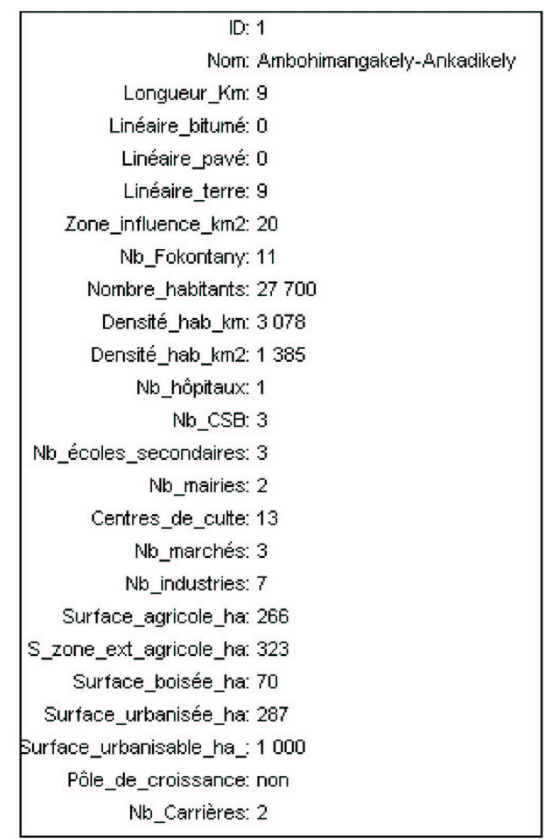

Figure 6: Extract of the data base information

Since the comparison between alternatives and profiles is based on a "weighted majority" rule the procedure for constructing such "weights" is the following (considering a set $H$ of $m$ criteria):

1. We first check if any majority of $m-1, m-2 \ldots$ criteria is sufficient to establish a preference relation (winning coalitions).

2. If all coalitions with $m / 2+1$ criteria are "winning" then we stop and consider that all criteria have the same importance and the rule is a simple majority rule.

3 . If it is not the case we identify the first $k$ such that not all coalitions with $m-k$ criteria are winning (obviously $k<m / 2$ ) and we ask which among these ones are winning and which not.

4. For each winning coalition $W \subset H$ we can write $\sum_{j \in W} w_{j} \geq \delta$ ( $\delta$ being the majority threshold, unknown).

5. We repeat the same procedure for $k+1, k+2$ until for some $k+l$ all coalitions are not winning.

6. At this point we have a set of linear inequalities, the variables being the different $w_{j}$ and $\delta$.

7. We fix an arbitrary value $K$ such that $\sum_{j \in H} w_{j}=K$. 


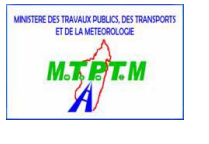

Tableau de performance

\begin{tabular}{|c|c|c|c|c|c|c|c}
\hline CRITERES & Axe 1 & Axe 2 & Axe 3 & Axe 4 & $\begin{array}{c}\text { Seuil } \\
\text { Profil : } \\
\text { axe à } \\
\text { financer } \\
\text { H1 }\end{array}$ & $\begin{array}{c}\text { Seuil } \\
\text { Profil : } \\
\text { axe à } \\
\text { négocier } \\
\text { H2 }\end{array}$ & \\
\hline C1 & 90 & 90 & 90 & 90 & 50 & $0-50$ & \\
\hline C2 & 10 & 10 & 10 & 30 & 40 & $0-40$ & \\
\hline C3 & B & A & A & M & A & L & \\
\hline C4 & 2 & 2 & 1 & 1 & 1 & 1 & \\
\hline C5 & 2500 & 5200 & 7000 & 2000 & 3000 & $2000-$ & 3000 \\
\hline C6 & 6 & 6 & 6 & 6 & 6 & $4-6$ & \\
\hline C7 & 15 & 4 & 3 & 2 & 14 & $14-20$ & \\
\hline
\end{tabular}

Figure 7: Data concerning the four alternatives and the profiles

8. We solve a linear programme minimising or maximising $\delta$ and we submit the result to the decision maker. In reality any feasible solution of the linear programme will fit, but the use of extreme solutions helps the decision maker to better discriminate among the values. For instance maximising $\delta$ we obtain a result which appears "highly confident" due to the qualified majority thus imposed. The reader should note that the arbitrary value $K$ is irrelevant for the model and only allows to find a numerical representation.

9. In case the decision maker is able to establish a partial order among the criteria this will translate in some further inequalities in the linear programme. It is also interesting to note that in case the decision maker makes any inconsistent statements these will result in an unfeasible linear programme.

The four alternatives have been compared to the two profiles. The first three have been classified as "to be financed", while the fourth one as "to be negotiated". The result has been discussed and validated with the public authorities concerned. The successful application of the procedure to the pilot study has been thus followed by introducing the method in the regular business conducted by AGETIPA. 


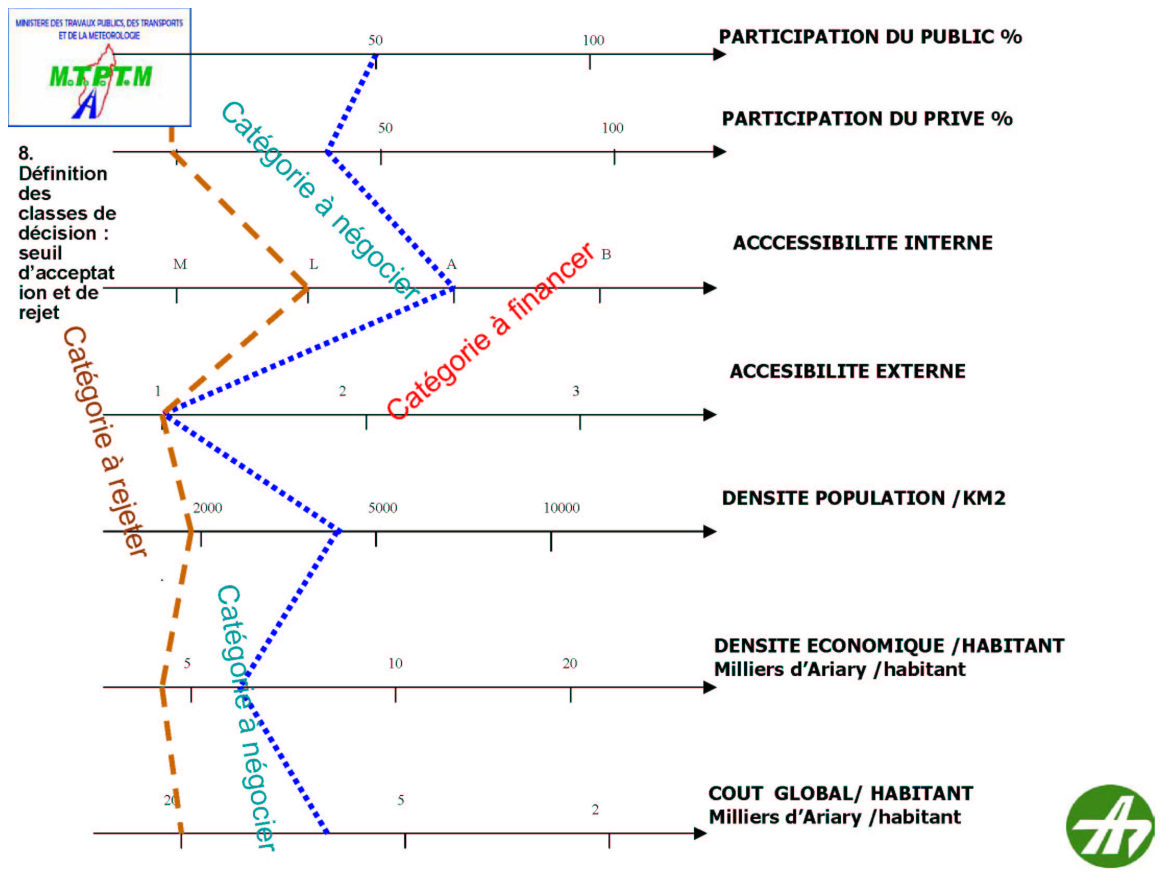

Figure 8: Classification model

\section{Feedback}

The method suggested in this document has been conceived in September 2006 and since then applied by AGETIPA together with the "engineering" teams working in rural road maintenance and other public works under the supervision of the World Bank and the government. In September 2008 there has been a follow up meeting involving expert engineers, the Public Work Minister and the World Bank.

An issue raised by the World Bank concerned the legitimation of the precise method adopted. Basically two questions have been raised:

- are we sure about the "correctness" of the results?

- why not using a "weighted sum" as any other does?

On the first question we offered the necessary literature references (such as [2], [7], [10], [13]). On the second question we emphasised the reasons for which the choice of establishing a single value function has been rejected: 1) the presence of information for which is not immediate establishing a trade-off among the criteria 
and 2) the necessity to have clear the reasons for which a project is not chosen (rejected or to negotiate) in order to pursue further participation of the local communities. It is interesting to notice however, the lack of information and training in evaluation models within an institution such as the Word Bank.

The engineers experts who applied the method in further experiences raised a number of questions concerning the definitions and the construction and the criteria. More precisely it has been discussed:

- The necessity to fix veto thresholds not in absolute value, but as a percentage of the criterion scale.

- The necessity to use attribute's information only once: in certain cases costs have been calculated as a cost by individual in the community concerned in order to compare a relative cost instead of an absolute one. However, this is an information already considered in the population density criterion. In order to avoid this problem it has been clarified to avoid such as a double use and prefer to establish a relative cost based on the length of the road to be maintained.

- The re-definition of the two criteria representing the commitment of the local community and the private actors. Most of the times it is unlike that any private actor can effectively contribute to the project. The result is that although in principle it is interesting to emphasise the presence of any such private actor, the criterion itself almost always never discriminates the alternatives. The suggestion (adopted) has been to consider the private actor's financial application as part of the whole involvement of the community and not separately. Instead it has been suggested, within the group, to separate the financial involvement of the local community to the short term direct costs of the project, from the further medium term maintenance costs.

A further element the discussion emphasised, was the necessity to establish a formal procedure, not only in order to apply, but also in order to further negotiate the application. The method as it is established today requires a procedure in order to formulate an application. Once the applicant knows that the proposal has been "approved" or "rejected" or that it is "negotiable", it is necessary to fix a procedure describing how these decisions are going to be implemented. For instance it is necessary to fix how the commitment of the local community to participate to the costs will be maintained after the decision to start the project has been taken (and the national financial commitment has been decided).

The overall assessment of the method has been positive. The projects handled using this approach show a better understanding, a major commitment of the local communities, while introducing accountability and elements of rationality in handling the road maintenance priorities. 


\section{Conclusions}

In the paper we present a real world decision support case: aiding AGETIPA (the operational agency of the Minister of Public Works in Madagascar) to handle the problem of "improving the rural road maintenance in Madagascar". As often happens behind the verbal presentation of the "problem" there exist multiple problem situations and problem formulations. In the document we present the ones fixed in this study and the reasons for doing so:

- take into account the qualitative information without necessarily transforming it in monetary consequences (not always feasible or accepted);

- allow and help to conduct negotiations with the local actors given the political nature of such decisions.

Technically the problem can be seen as an "ordinal measurement" problem where to each object to be measured is associated a label from an ordinal scale (in other terms a class among a set of ordered classes; in this case accepted, negotiable, rejected). A further specificity results from the existence of a hierarchy of similar evaluation models where once again the objects to be evaluated must be classified in ordered classes (for instance level of service 1, 2, 3). The overall assessment resulting from the hierarchical aggregation of the values (assignment to ordered classes) obtained on the nodes of the evaluation tree.

The model has been first applied with a pilot case study (in the Metropolitan Area of the Great Antananarivo, reported in this document) and then used for the last two years. We briefly report the feedback received from this experience. AGETIPA and the teams of engineers working in this area are satisfied with the methodology. Presently AGETIPA is considering the extension of the use of decision aiding methodology in other case studies including the location of a new

port, the construction of new schools and the renovation of the "pedestrian path network" within Antananarivo.

\section{References}

[1] A. Boardman. Cost benefit analysis: Concepts and practices. Prentice-Hall, New-York, 1996.

[2] D. Bouyssou, T. Marchant, M. Pirlot, P. Perny, A. Tsoukiàs, and Ph. Vincke. Evaluation and decision models: a critical perspective. Kluwer Academic, Dordrecht, 2000. 
[3] João N. Clímaco and Luis C. Dias. An approach to support negotiation processes with imprecise information multicriteria additive models. Group Decision and Negotiation, 15:171 - 184, 2006.

[4] P. Dasgupta and D. Heal. Economic Theory and Exhaustible Resources. Cambridge University Press, Cambridge, 1979.

[5] C. Dinwiddy and F. Teal. Principles of cost-benefit analysis for developing countries. Cambridge University Press, Cambridge, 1996.

[6] R.L. Keeney. Value-Focused Thinking. A Path to Creative Decision Making. Harvard University Press, Cambridge, 1992.

[7] R.L. Keeney and H. Raiffa. Decisions with multiple objectives: Preferences and value tradeoffs. J. Wiley, New York, 1976.

[8] R.B. Kulkarni, D. Miller, R.M. Ingram, C-W. Wong, and J. Lorenz. Needbased project prioritization: Alternative to cost-benefit analysis. Journal of Transportation Engineering, 130:150 - 158, 2004.

[9] J. Liesiö, P. Mild, and A. Salo. Preference programming for robust portfolio modeling and project selection. European Journal of Operational Research, 181:1488 - 1505, 2007.

[10] L.Y. Maystre, J. Pictet, and J. Simos. Méthodes multicritères ELECTRE. Description, conseils pratiques et cas d'application à la gestion environnementale. Presses Polytechniques et Universitaires Romandes, Lausanne, 1994.

[11] P. Mild and A. Salo. Combining a multiattribute value function with an optimization model: An application to dynamic resource allocation for infrastructure maintenance. Decision Analysis, 6:139 - 152, 2009.

[12] V. Mousseau, R. Slowinski, and P. Zielniewicz. A user-oriented implementation of the ELECTRE TRI method integrating preference elicitation support. Computers \& Operations Research, 27(7-8):757-777, 2000.

[13] J. Pictet and D. Bollinger. Adjuger un marché au mieux-disant. Analyse multicritère, pratique et droit des marchés publics. Presses Polytechniques et Universitaires Romandes, Lausanne, 2003. 
[14] B. Roy and S. Damart. L'analyse coûts-avantages, outil de concertation et de légitimation? METROPOLIS, 108-109:7-16, 2002.

[15] A. Tsoukiàs. On the concept of decision aiding process. Annals of Operations Research, 154:3 - 27, 2007.

[16] Ph. Vincke. Multicriteria Decision-Aid. J. Wiley, New York, 1992.

\section{Appendix A}

The basic concepts adopted in the procedure used (based on ELECTRE TRI) are the following.

- A set $A$ of alternatives $a_{i}, i=1 \cdots m$.

- A set $G$ of criteria $g_{j}, j=1 \cdots n$. A relative importance $w_{j}$ (usually normalised in the interval $[0,1])$ is attributed to each criterion $g_{j}$.

- Each criterion $g_{j}$ is equipped with an ordinal scale $\mathcal{E}_{j}$ with degrees $e_{j}^{l}, l=$ $1 \cdots k$.

- A set $\mathcal{P}$ of profiles $p_{h}, h=1 \cdots t, p_{h}$ being a collection of degrees, $p_{h}=$ $\left\langle e_{1}^{h} \cdots e_{n}^{h}\right\rangle$, such that if $e_{j}^{h}$ belongs to profile $p_{h}, e_{j}^{h+1}$ cannot belong to profile $p_{h-1}$.

- A set $\mathcal{C}$ of categories $c_{\lambda}, \lambda=1 \cdots t+1$, such that the profile $p_{h}$ is the upper bound of category $c_{h}$ and the lower bound of category $c_{h+1}$.

- An outranking relation $S \subset(A \times \mathcal{P}) \cup(\mathcal{P} \times A)$, where $s(x, y)$ should be read as " $x$ is at least as good as $y$ ".

- A set of preference relations $\left\langle P_{j}, I_{j}\right\rangle$ for each criterion $g_{j}$ such that:

- $\forall x \in A P_{j}\left(x, e_{j}^{h}\right) \Leftrightarrow g_{j}(x) \succ e_{j}^{h}$

$-\forall x \in A P_{j}\left(e_{j}^{h}, x\right) \Leftrightarrow g_{j}(x) \prec e_{j}^{h}$

$-\forall x \in A I_{j}\left(x, e_{j}^{h}\right) \Leftrightarrow g_{j}(x) \approx e_{j}^{h}$

$\prec, \approx$ induced by the ordinal scale associated to criterion $g_{j}$.

The procedure works in two basic steps. 
1. Establish the outranking relation on the basis of the following rule:

$$
s(x, y) \Leftrightarrow C(x, y) \text { and not } D(x, y)
$$

where

$$
\begin{gathered}
\forall x \in A, y \in \mathcal{P}: C(x, y) \Leftrightarrow \sum_{j \in G^{ \pm}} w_{j} \geq c \text { and }\left(\sum_{j \in G^{+}} w_{j} \geq \sum_{j \in G^{-}} w_{j}\right) \\
\forall y \in A, x \in \mathcal{P}: C(x, y) \Leftrightarrow \\
\left(\sum_{j \in G^{ \pm}} w_{j} \geq c \text { and } \sum_{j \in G^{+}} w_{j} \geq \sum_{j \in G^{-}} w_{j}\right) \text { or }\left(\sum_{j \in G^{+}} w_{j}>\sum_{j \in G^{-}} w_{j}\right) \\
\forall(x, y) \in(A \times \mathcal{P}) \cup(\mathcal{P} \times A): \operatorname{not} D(x, y) \Leftrightarrow \\
\sum_{j \in G^{-}} w_{j} \leq d \text { and } \forall g_{j} \text { not } v_{j}(x, y)
\end{gathered}
$$

where

- $G^{+}=\left\{g_{j} \in G: P_{j}(x, y)\right\}$

- $G^{-}=\left\{g_{j} \in G: P_{j}(y, x)\right\}$

- $G^{=}=\left\{g_{j} \in G: I_{j}(x, y)\right\}$

- $G^{ \pm}=G^{+} \cup G^{=}$

- $c$ : the concordance threshold $c \in[0.5,1]$

- $d$ : the discordance threshold $d \in[0,1]$

- $v_{j}(x, y)$ : veto, expressed on criterion $g_{j}$, of $y$ on $x$

2. When the relation $S$ is established, assign any element $a_{i}$ on the basis of the following rules.

\section{1 pessimistic assignment}

- $a_{i}$ is iteratively compared with $p_{t} \cdots p_{1}$,

- as soon as $s\left(a_{i}, p_{h}\right)$ is established, assign $a_{i}$ to category $c_{h}$. 


\section{2 optimistic assignment}

- $a_{i}$ is iteratively compared with $p_{1} \cdots p_{t}$,

- as soon as is established $s\left(p_{h}, a_{i}\right) \wedge \neg s\left(a_{i}, p_{h}\right)$ then assign $a_{i}$ to category $c_{h-1}$.

The pessimistic procedure finds the highest profile for which the element is not worse. The optimistic procedure finds the lowest profile against which the element is surely worse. If the optimistic and pessimistic assignments coincide, then no uncertainty exists for the assignment. Otherwise, an uncertainty exists and should be considered by the user. 